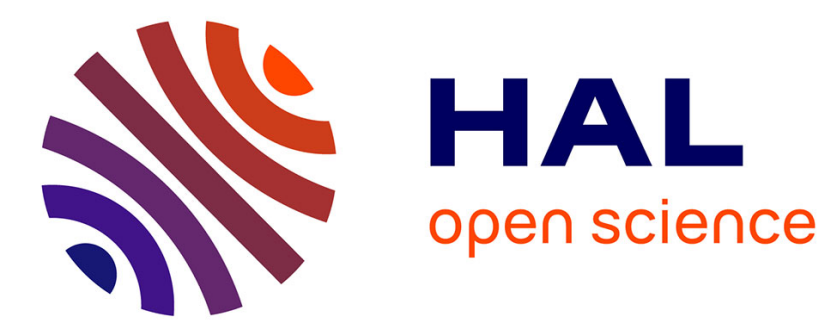

\title{
XANES AND THE DETERMINATION OF BOND ANGLES
}

\author{
J. Pendry
}

\section{To cite this version:}

J. Pendry. XANES AND THE DETERMINATION OF BOND ANGLES. Journal de Physique Colloques, 1985, 46 (C9), pp.C9-93-C9-100. 10.1051/jphyscol:1985911 • jpa-00225272

\section{HAL Id: jpa-00225272 https://hal.science/jpa-00225272}

Submitted on 1 Jan 1985

HAL is a multi-disciplinary open access archive for the deposit and dissemination of scientific research documents, whether they are published or not. The documents may come from teaching and research institutions in France or abroad, or from public or private research centers.
L'archive ouverte pluridisciplinaire HAL, est destinée au dépôt et à la diffusion de documents scientifiques de niveau recherche, publiés ou non, émanant des établissements d'enseignement et de recherche français ou étrangers, des laboratoires publics ou privés. 
JOURNAL DE PHYSIQUE

Colloque C9, supplément au $n^{\circ} 12$, Tome 46, décembre 1985

page C9-93

XANES AND THE DETERMINATION OF BOND ANGLES

J.B. Pendry

The Blackett Laboratory, Imperial College, London SW7 2BZ, U.K.

\begin{abstract}
It is argued that conventional weak scattering probes provide no direct information beyond the radial distribution function. In contrast, X-ray Absorption Near Edge Structure is produced by the multiple scattering of low energy electrons and has sensitivity to bond angles and symmetry of the environment
\end{abstract}

\title{
I. WEAK SCATTERING AND PAIR CORRELATIONS
}

The traditional probe of structure is a wave that is weakly scattered by the material under consideration, such as $\mathrm{X}$-rays or neutrons. Weak scattering has the great virtue of a simple interpretation. For example, a plane wave,

$$
\exp \left(\underline{k}_{i} \cdot \underline{r}\right),
$$

incident on the solid is scattered to an outgoing wave,

$$
A(\underline{g}) \exp \left(\underline{k}_{f} \cdot \underline{r}\right) \text {, }
$$

where

$$
\begin{aligned}
A(q) & =\Sigma f_{s}(q) \exp \left(i g \cdot \underline{R}_{S}\right), \\
g & =\underline{k}_{i}-\underline{k}_{f},
\end{aligned}
$$

and $\underline{R}_{s}$ is the position of the $s$ th atom, with scattering factor $f_{s} ; \underline{k}_{i}$ and $\underline{k}_{f}$ are the incident and scattered wave vectors respectively. The experiment observes

$$
|A(g)|^{2}=\Sigma E_{s} F_{t}^{*} \exp \left(i g \cdot\left(\underline{R}_{S}-\underline{R}_{t}\right)\right),
$$

an expression in which the atomic coordinates occur in pairs, and only in pairs. Such an expression can only ever tell us about the pair correlation function in the material but in ordered, crystalline materials this information is usually sufficient to give us the higher order correlation functions by implication $/ 1 / 2 /$. In the case of an orientated crystal measurement of $|A|^{2}$ for a full set 
of $3 \mathrm{D}$ vectors, $q$, provides enough information to fix the higher order correlations. By contrast, in a liquid there is much less information, available because the scattering is on average isotropic so that $\left.|A|\right|^{2}$ is a function only of $|\mathrm{g}|$. Occasionally this provides enough information to determine all correlation functions, but in most instances this is not the case. In this sense disordered materials differ in a fundamental way from their ordered counterparts. Their structure is much more subtle, as indicated by their finite entropy content, and demands new probes to reveal its details to us.

\section{MULTIPLE SCATTERING - 1 PROBLEM FOR THE THEORIST}

When scattering is strong, the probe can be expected to interact with more than one scattering centre in the solid, and equation (3) must be generalised to

$$
\begin{aligned}
A_{m s}\left(\underline{k}_{f}, \underline{k}_{i}\right)=\Sigma & \exp \left(i \underline{k}_{f} \cdot \underline{R}_{s}\right) E_{s}\left(\underline{k}_{f}, \underline{k}_{i}\right) \exp \left(i \underline{k}_{i} \cdot \underline{R}_{s}\right)+ \\
& \exp \left(i \underline{k}_{f} \cdot \underline{R}_{s}\right) f_{s} G_{s t} f_{t} \exp \left(i \underline{k}_{i} \cdot \underline{R}_{t}\right)+ \\
& \exp \left(i \underline{k}_{f} \cdot \underline{R}_{s}\right) f_{s} G_{s u} f_{u} G_{u t} f_{t} \exp \left(i \underline{k}_{i} \cdot \underline{R}_{t}\right) \\
& \text { (plus higher order terms) }
\end{aligned}
$$

The new ingredients in this formula are

$$
\begin{aligned}
& \text { the scattering factors, f, are now generally } \\
& \text { complex, reflecting multiple scattering events } \\
& \text { within individual atoms. }
\end{aligned}
$$

The scattered amplitude, $A_{m s}$, is no longer a function of $\left|k_{f}-\underline{k}_{i}\right|$ alone, ${ }_{i}$ independent depends on $\underline{k}_{f}$ and

Several atomic coordinates appear in a typical term through the propagators, $G$, which represent the wave travelling between atoms.

This last point becomes more explicit if we take the case of point scatterers for which

$$
\left.G_{s t}=\exp \left(i k \mid \underline{R}_{s}-\underline{R}_{t}\right) \mid\right)
$$

so that the overall dependence on atomic coordinates of the last term in equation ( 6 ) would be

$$
\exp \left(i \underline{k}_{f} \cdot \underline{R}_{s}+i k\left|\underline{R}_{s}-\underline{R}_{u}\right|+i k\left|\underline{R}_{u}-\underline{R}_{t}\right|+i \underline{k}_{i} \cdot \underline{R}_{t}\right) \text {. }
$$

As the wave explores the solid, scattering from successive atoms, it measures the path length through the phase which it acquires. We might imagine that in principle we could find all possible path lengths for multiple scatterings in the medium by Fourier transforming the scattered intensities. This information could be interpreted in terms of pair correlations. However the program is not a practicable one, partly because the propagators, $G$, are rarely simple phase factors, and partly because the individual atomic scattering factors, $f$, introduce further complications which are difficult to correct for. 
The only practical way of proceeding that has been used to interpret multiple scattering data has been to postulate a trial structure, calculate the implied spectrum, compare with experiment, and then successively refine the structure. The passage from trial structure to calculated spectrum is non-trivial and is one of the limiting factors to interpretation of data which in principle contain the information we require.

one multiple scattering probe that has been much used in structure determination is the low energy electron/3/. Traditionally low energy electron diffraction (LEED) has been used as a surface structure probe. Here the strong scattering interaction is required to give surface sensitivity, and the multiple scattering processes are regarded as something of a nuisance. LEED experiments are now routinely interpreted to give surface structures, and essentially the same theory can be used in another context to give higher order correlation functions.

$X$-ray absorption near edge structure (XANES, or NEXAFS as some call it) $/ 1 / 4 /$ results from the ejection of an electron from an inner core of the absorbing atom. The ability of the electron to escape from the atom determines the absorption cross section and in the near edge region, where the electron has, say, less than $50 \mathrm{eV}$ of kinetic energy, multiple scattering is dominant; in contrast to the region of extended X-ray absorption fine structure (EXAFS) /5/6/, more than $50 \mathrm{eV}$ above the edge, in which cross sections are smali enough for most multiple scattering processes to be neglected. Here we have a probe which can be tuned to the environment of a particular atom by selecting the appropriate absorption edge. The ejected electron having a finite elastic lifetime explores only the immediate surroundings of that atom before it vanishes in incoherent processes which in turn limits the number of multiple scattering events that we have to interpret.

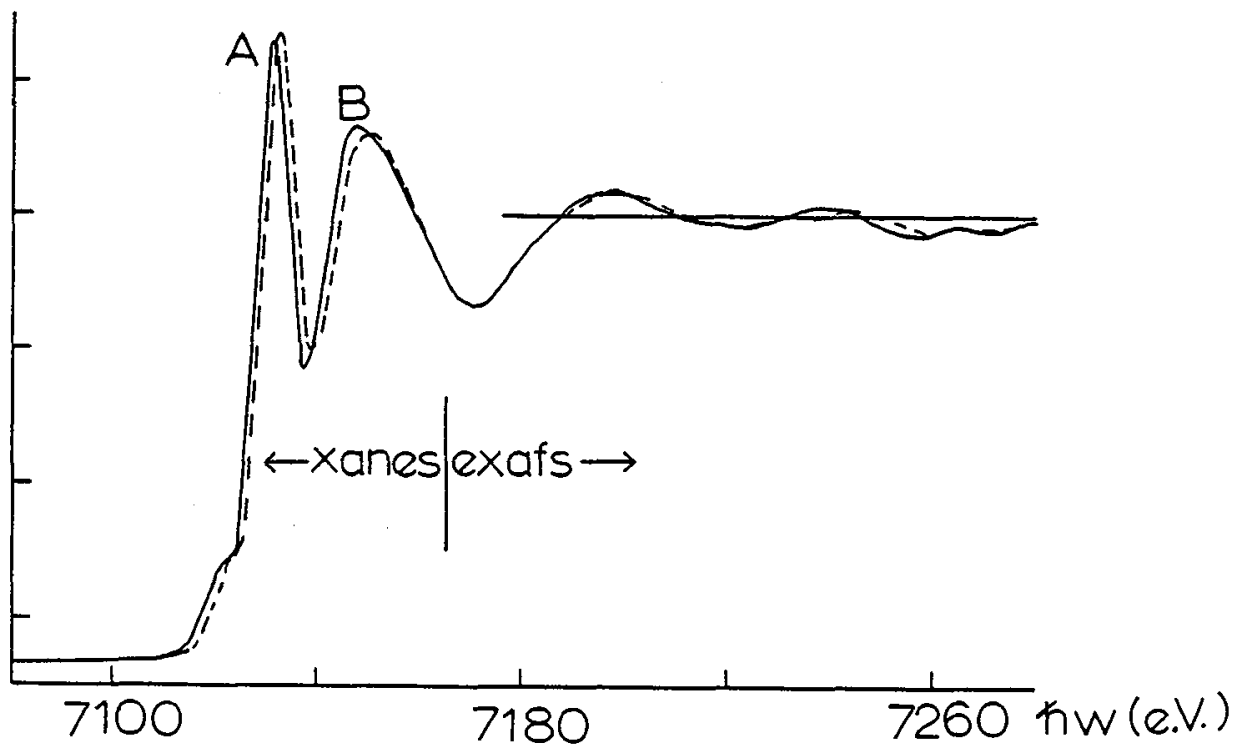

Fig. 1 - The relative absorption coefficient for x-rays in the vicinity of the iron $k$ edge. The division of the spectrum into XANES and EXAFS is somewhat arbitrary. $\mathrm{K}_{4} \mathrm{Fe}(\mathrm{CN})_{6} \cdot 3 \mathrm{H}_{2} \mathrm{O}$ and $\ldots-\mathrm{K}_{3} \mathrm{Fe}(\mathrm{CN})_{6}$. 
Figure 1 shows the $X$-ray absorption spectrum for an iron $K$ edge in $\mathrm{K}_{3} \mathrm{Fe}(\mathrm{CN}) \mathrm{f} / 8 \%$. Clearly the first $50 \mathrm{eV}$ of the spectrum is heavily modulated by scattering of the ejected electron and does in fact contain information beyond the radial distribution function.

Despite the complexity of the multiple scattering process some physical insight into how such quantities as bond angles affect spectra is possible. This is particularly true at the limits of the XANES energy range where strong scattering of electrons is largely confined to the forward direction. In this case the single scattering spectrum is strongly perturbed when the central emitting atom and two further atoms lie in a straight line. The central atom gets in the way of signals returning from the furthest atom and a single scattering interpretation of the spectrum under these circumstances leads to erroneous bond lengths. Sometimes the effect is referred to as "shadowing" by the central atom, though a more accurate description is "focussing" because the attractive potential around the atom draws in the electrons and enhances their intensity at the furthest atom.

At lower energies within 5 or $10 \mathrm{eV}$ of threshold another rather different picture is sometimes more appropriate. The states in this energy range can be thought of as antibonding states which reflect the chemistry of the environment. Thus it is not surprising if bond angles (ie three - body correlation functions) have a strong effect on spectra. We can expect sensitivity to such chemically relevant quantities as the symmetry of the environment as well as to saturation of bonds.

\section{SOME MULTIPLE SCATTERING CALCULATIONS}

We shall not dwell here on details of how the calculations are performed which is described in detail in the literature/1/9/10/. It is possible to describe the scattering of the individual atoms by spherically symmetric one - electron potentials constructed according to one of the standard "muffin - tin" procedure, using the local density formalism. The multiple scattering between atoms is a more difficult quantity to handle as explicit summation of the terms of various orders in equation (6) is not possible, even if the series were to converge. By grouping the terms in a systematic fashion calculations can be made for quite complicated trial geometries, especially if there is any symmetry to be exploited. Even for situations without much symmetry it is now possible to make calculations, thanks to the development of new, fast, perturbation schemes/11/12/13/. The calculations require a large computer such as the Cray, but are probably much less expensive than the experiments they are designed to interpret.

To verify that experiments can be reproduced by theory when the geometry is known we show in figure 2 some calculations for silver, taken from reference $/ 1 /$. To describe the data it was necessary to consider a cluster of 43 atoms centred about the absorbing atom. The main features of the spectrum are reproduced, especially the positions of peaks which are especially significant because of the implication that we have got the conditions for constructive interference right, and hence have the right bond lengths and angles in our calculation. The intensities are recognised to be more subtle quantities. 


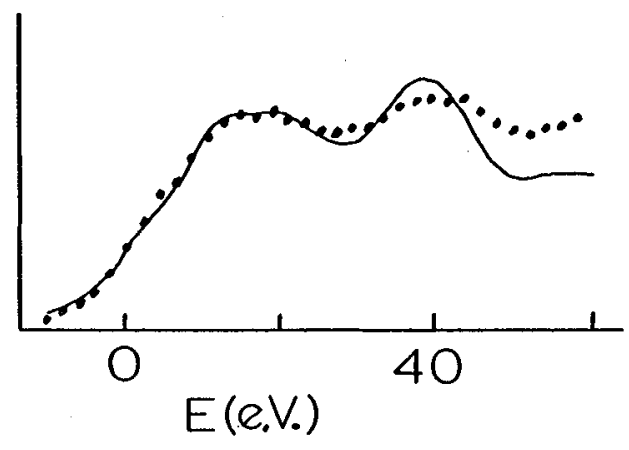

Fig. 2 - K-edge XANES for silver. The calculations (full curve) used a 3-shell, 43 atom, cluster. The experimental (dashed) curve was taken from reference $/ 14 /$.

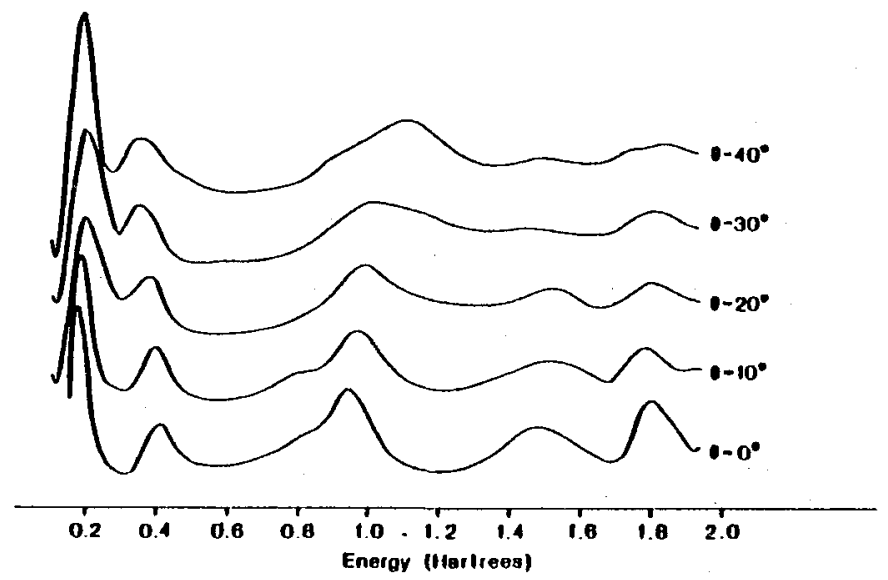

Fig. 3 - K-edge XANES calculation for the transition metal carbonyl compound for various angles of rotation $(\Theta)$ of the oxygen sheli.

To test how sensitive data are likely to be to bond angles a hypothetical carbonyl compound was investigated. It comprised a central transition metal octagonally coordinated by six carbonyl groups. In most compounds of this type the metal - carbon and carbon - oxygen bonds lie in the same straight line, but can occasionally be bent. The test calculation examined changes in the spectra for geometries in which the radial distribution about the central atom was preserved. Imagine the $z$-axis chosen along one of the 
metal-carbonyl bonds. In the $x-y$ plane there are two rings: an inner one consisting of four carbon atoms, and an outer one consisting of four oxygen atoms. The outer ring can be rotated, preserving the radial distribution as required, but changing the carbon-oxygen bond angle. In any weak - scattering theory such rotations would not change the spectra. Any changes are therefore entirely and exclusively attributable to the changes in bond angle. Figure 3 shows the results for various angles of rotation and indicates that there is substantial sensitivity to the angle.
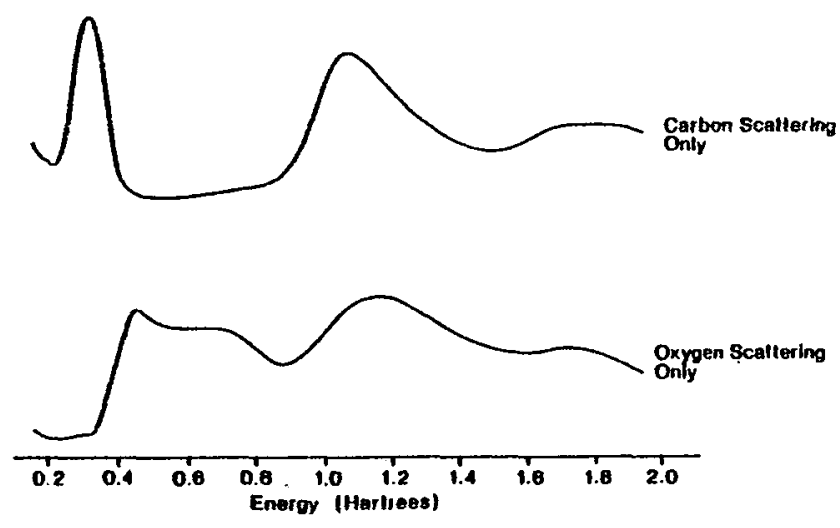

Fig. 4 - Calculations as above for $\Theta=0$ but with scattering from only the carbon, or oxygen shells included.

In figure 4 we show the spectra when only carbon and only oxygen are present in the system to make the point that the spectrum for the carbonyl is not simply the sum of these two spectra, as would be the case in a single scattering theory.

Finally to illustrate that the calculations can be performed for systems having considerable complexity, figure 5 shows some calculations for haemoglobin compared to experiment $/ 15 /$. The cluster used in the calculations consisted of the porphyrin group together with the imidazole group. The porphyrin group was idealised in that it was taken to be planar and four-fold symmetrical, though in more recent versions of the theory this idealisation would probably not be necessary. The agreement with experiment is seen to be very encouraging. 


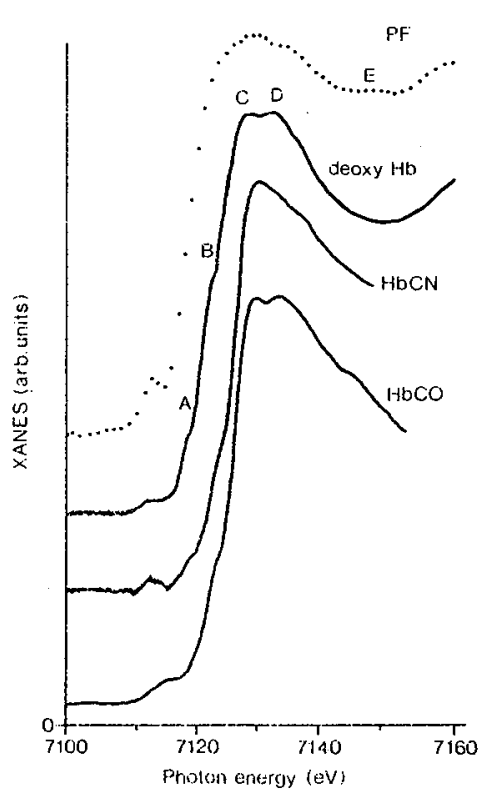

experiment

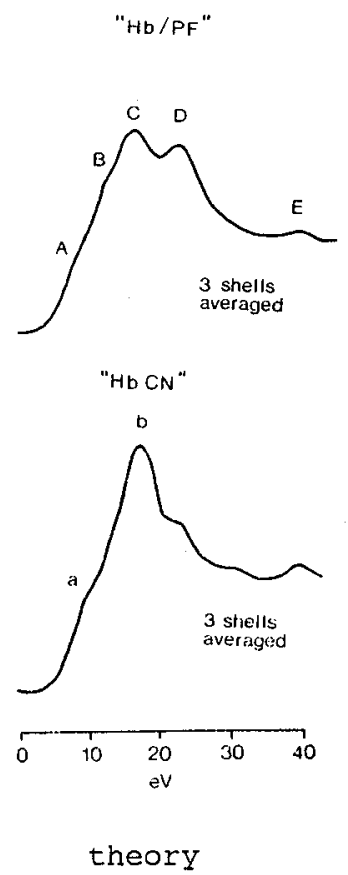

theory

Fig. 5 - Experimental K-edge XANES for the picket fence compound PF, deoxy $\mathrm{Hb}, \mathrm{HbCN}$ and $\mathrm{HbCO}$. Theoretical curves for the picket fence compound and for $\mathrm{HbCN}$ are also shown.

\section{CONCLUSIONS}

Whilst being optimistic about the potential of XANES to provide information beyond the radial distribution function, it should be said that there are limitations to the technique. The chief of these probably lies in the experimental data themselves. They suffer from an inherent limitation to the resolution in energy dictated by the finite lifetime of the core hole being created. Structure in spectra on a scale finer than about $3 \mathrm{eV}$ is not observed. Given that the range over which multiple scattering effects are prominent is only about $50 \mathrm{eV}$, it can be seen that the number of independent pieces of information contained in the data is of the order of 10 . Thus to imagine that a XANES experiment would find all the bond angles in a complex amorphous system is a hopeless dream. A more realistic aim would be to start from a situation in which something is already known about the system, perhaps from chemical arguments, and to ask the experiments to distinguish between perhaps two or three clearly defined models, either by making use of theoretical calculations predicting their XANES spectra, or by making comparisons with spectra taken for model compounds of known structure. Within these limitations XANES can provide us with information that is available from no other source. 


\section{REFERENCES}

/1/ Durham P.J., Pendry J.B., and Hodges C.H., Sol st Comm 38 (1981) 159.

12/ Pendry J.B., Comments on Solid State Physics 10 (1982) 1

/3/ Pendry J.B., "Low Energy Electron Diffraction" (Academic, London $1974)$

/4/ Belli M., Scafati A., Bianconi A., Mobilio S., Palladino L., Reale A., Burattini E. Sol. St. Comm. 35 (1980) 355 .

/5/ Sayers D. E., Stern E. A., Lytle F. W. Phys. Rev. Lett. 27 (1971) 1204 .

/6/ Lee P. A. and Pendry J. B. Phys. Rev. B11 (1975) 2795.

/7/ Stohr J., Jaeger R., and Brennan S. Surf. Sci. 117 (1982) 503.

/8/ Bianconi A., Dell'Ariccia M., Durham P. J., and Pendry J. B. Phys. Rev. B26 (1982) 6502 .

/9/ Natoli C. R., Misemer D. K., Doniach S., and Kutzler F. W. Phys. Rev. A.22 (1980) 1104 .

/10/ Durham P. J., and Pendry J. B., and Hodges C. H. Comp. Phys. Comm. 25 (1982) 193.

/11/ Pendry J. B. J. Phys. C4 (1971) 3095.

$112 /$ Rehr J. J., and Stern E. A. Phys. Rev. B14 (1976) 4413.

/13/ Vvedensky D. D., and Pendry J. B. Surf. Sci. 152 (1985) 33.

/14/ Muller J. E., Jepsen O., Anderson 0 . K., and Wilkins J. W.

Phys. Rev. Lett. 40 (1978) 720 .

/15/ Durham P. J., Hasnain S. S., Bianconi A., Congiu-Castellano A., Giovanelli A., Incoccia L., Morante S., and Pendry J. B. The EMBO Journal $\underline{2}$ (1983) 1441 . 